\title{
Feasibility of near-infrared spectroscopic tomography for intraoperative functional cerebral monitoring: A primate study
}

\author{
Daniel C. Lee, MD, ${ }^{\text {a,b,c }}$ Tigran Gevorgyan, $\mathrm{MD},{ }^{\mathrm{c}}$ Harry L. Graber, PhD ${ }^{\mathrm{d}}$ Douglas S. Pfeil, PhD, \\ Yong $\mathrm{Xu}, \mathrm{PhD},{ }^{\mathrm{d}}$ Sundeep Mangla, MD, ${ }^{\mathrm{f}, \mathrm{g} h}$ Frank C. Barone, PhD, ${ }^{\mathrm{h}, \mathrm{i}}$ Jenny Libien, MD, PhD, ${ }^{\mathrm{e}, \mathrm{h}}$ \\ Jean Charchaflieh, MD, MPH, DrPH, ${ }^{\mathrm{j}}$ John G. Kral, MD, PhD, ${ }^{\mathrm{k}}$ Sergio A. Ramirez, MD, \\ LeRone Simpson, MD, ${ }^{\mathrm{m}}$ and Randall L. Barbour, $\mathrm{PhD}^{\mathrm{d}, \mathrm{e}}$
}

\begin{abstract}
Objective: The wide-ranging manipulations to the cardiovascular system that frequently occur during cardiac surgery can expose the brain to variations in its blood supply that could prove deleterious. As a first step to developing a resource suitable for monitoring such changes, we detected the hemodynamic events induced in the brain of a primate model, using high-density near-infrared spectroscopy combined with tomographic reconstruction methods and validated the findings using established radiologic and histologic techniques.
\end{abstract}

\begin{abstract}
Methods: Continuous monitoring of the relative changes in the components of the cerebral hemoglobin signal was performed using high-density near-infrared spectroscopy (270 source-detector channel array) in anesthetized bonnet macaques with the brain exposed to induced ischemia and other acute events. A comparative analysis (exact binomial test) applied to reconstructed 3-dimensional images before and after the events and between cerebral hemispheres, combined with postprocedure magnetic resonance imaging, and postmortem histopathologic examination of the macaques' brains was performed to document and validate the spatial features revealed by the optical findings.
\end{abstract}

Results: Relative changes in the measured and calculated components of the hemoglobin signal, in response to the performed manipulations, revealed substantial concurrence among the reconstructed 3-dimensional images, magnetic resonance imaging of the macaques' brains, and postmortem histopathologic examination findings. Concurrence was seen when the manipulated hemoglobin concentration and associated oxygenation levels were either increased or decreased, and whether they were bilateral or restricted to a specified hemisphere.

Conclusions: Continuous near-infrared spectroscopy tomography has been shown to accurately capture and localize cerebral ischemia, vasodilatation, and hemorrhage in primates in real time. These findings are directly applicable to clinical intraoperative functional cerebral monitoring. (J Thorac Cardiovasc Surg 2014;148:3204-10)

From the Section of Thoracic/Cardiovascular Surgery, ${ }^{a}$ University of Oklahoma College of Medicine, Oklahoma City, Okla; Joint Program in Biomedical Engineering, ${ }^{\text {b }}$ State University of New York, Downstate School of Graduate Studies and New York University Polytechnic School of Engineering, Brooklyn, NY; Department of Medicine, ${ }^{\mathrm{c}}$ Interfaith Medical Center, Brooklyn, NY; NIRx Medical Technologies, LLC, ${ }^{\mathrm{d}}$ Los Angeles, Calif; Departments of Pathology, ${ }^{\mathrm{e}}$ Radiology, ${ }^{\mathrm{f}}$ Neurosurgery, ${ }^{\mathrm{g}}$ Neurology, ${ }^{\mathrm{h}}$ Physiology and Pharmacology, ${ }^{\mathrm{i}}$ and Surgery, ${ }^{\mathrm{k}}$ State University of New York, Downstate Medical Center, Brooklyn, NY; Department of Anesthesiology, ${ }^{j}$ Yale University School of Medicine, New Haven, Conn; Department of Surgery, ${ }^{1}$ Montefiore New Rochelle Hospital, New Rochelle, NY; and Department of Surgery, ${ }^{\mathrm{m}}$ Texas Tech University Health Science Center, Lubbock, Tex.

This research was support by the National Institutes of Health (grants R21NS067278, R42NS050007, and 5R44NS049734); the Defense Advanced Research Projects Agency project (grant N66001-10-C-2008); and the New York State Department of Health to Drs Barbour and Lee.

Disclosures: Harry L. Graber and Yong Xu are employees of NIRx Medical Technologies LLC. Randall L. Barbour is the CEO of NIRx Medical Technologies LLC. The other authors have nothing to disclose with regard to commercial support.

Read at the 94th Annual Meeting of The American Association for Thoracic Surgery, Toronto, Ontario Canada, April 26-30, 2014.

Received for publication April 27, 2014; revisions received July 2, 2014; accepted for publication July 13, 2014; available ahead of print Oct 17, 2014.

Address for reprints: Daniel C. Lee, MD, Section of Thoracic/Cardiovascular Surgery, University of Oklahoma College of Medicine, 920 Stanton L. Young Blvd, WP2230, Oklahoma City, OK 73104 (E-mail: Daniel-C-Lee@ ouhsc.edu).

0022-5223/\$36.00

Copyright (c) 2014 by The American Association for Thoracic Surgery

http://dx.doi.org/10.1016/j.jtcvs.2014.07.041

See related commentary on pages 3211-2.

Supplemental material is available online.

Approximately 400,000 coronary artery bypass grafting and 100,000 valve procedures are performed annually in the United States, with an average hospital charge of $\$ 150,000 /$ case and $\$ 200,000 /$ case, respectively. ${ }^{1}$ Despite major advances during the past 4 decades that have served to reduce nearly all complications associated with cardiac surgery, neurologic complications have remained unabated. ${ }^{2}$ Central nervous system injuries range from debilitating or lethal stroke to encephalopathy, delirium, and neurocognitive decline. These complications have significant clinical and economic implications in perioperative mortality, ${ }^{3}$ length of hospital stay, and resource usage. ${ }^{4}$ Neurocognitive dysfunction was noted to affect $50 \%$ of patients undergoing coronary artery bypass grafting at discharge and is predictive of long-term deficit, ${ }^{5}$ leading to a decline in patients' quality of life. ${ }^{6,7}$ 


$$
\begin{aligned}
& \text { Abbreviations and Acronyms } \\
& \triangle \mathrm{COE}=\text { cerebral oxygen exchange } \\
& \text { fNIRS = functional near-infrared spectroscopy } \\
& \mathrm{Hb}=\text { hemoglobin } \\
& \mathrm{Hb}_{\text {deoxy }}=\text { deoxyhemoglobin } \\
& \mathrm{Hb}_{\text {oxy }}=\text { oxyhemoglobin } \\
& \mathrm{Hb}_{\text {total }}=\text { total hemoglobin } \\
& \text { IV }=\text { intravenous } \\
& \text { MCA = middle cerebral artery } \\
& \text { NIRS = near-infrared spectroscopy } \\
& \text { SAH }=\text { subarachnoid hemorrhage }
\end{aligned}
$$

Strategies for predicting and forestalling dysfunction would favorably affect the cost of care and maximize improvements in the quality of life afforded by cardiac surgery. Access to techniques that support intraoperative cerebral monitoring is an important factor guiding the development of improved protective strategies.

The currently available intraoperative cerebral monitoring modalities are suboptimal. Strategies for monitoring hemodynamic status include invasive measures of intracranial pressure or regional flow using implanted thermal diffusion microprobes, which carry added risk. ${ }^{8}$ Noninvasive transcranial Doppler monitors high-intensity transient signals, and the number of emboli thus detected can correlate with the neurocognitive outcome. ${ }^{9}$ However, problems have been encountered with reproducibility, and the sensitivity of this method is limited by the cranium. ${ }^{9,10}$ Electrical activity of the brain can be monitored noninvasively using electroencephalography, but this requires the intraoperative presence of a neurologist, incurring added cost. Furthermore, the observed changes reflect only the activity of the cortex (not subcortex), and they are often influenced by the anesthesia and hypothermia rather than by hypoperfusion. ${ }^{9}$

Brain oxygenation can be monitored invasively using microelectrodes inserted into the white matter ${ }^{11}$ or by measuring the jugular venous oxygen saturation, although these methods have associated risks. ${ }^{12}$ Approved noninvasive measures include the use of low-density (an array of $<10$ source-detector pairings [channels]) near-infrared spectroscopic (NIRS) oximetry, which serves to measure relative trends in regional oxygen saturation (eg, INVOS ${ }^{13}$; Covidien, Dublin, Ireland) or, purportedly, absolute cerebral tissue oxygen saturation (FORE-SIGHT ${ }^{14}$; CASMED, Branford, Conn). Although these techniques can sensitively detect regional changes in cerebral oxygenation, they are unable to determine the spatial heterogeneity of cerebral perfusion occurring over large tissue volumes ${ }^{15}$ and are limited in their analysis methods owing to their small, low-density array design.

An ideal intraoperative cerebral monitoring tool should provide real-time information about brain oxygenation, with temporal and spatial specificity, and should identify ischemia and hypoperfusion at a reversible stage to allow countermeasures by surgeons and anesthesiologists. High-density NIRS tomography is a portable, noninvasive functional brain imaging modality without the abovedescribed limitations. Furthermore, it has been used in many experimental and preclinical studies. ${ }^{16} \mathrm{We}$ designed a controlled cerebral ischemia study with additional manipulations, using a nonhuman primate (bonnet macaque), to validate the capability of NIRS tomography to monitor relevant clinical events.

\section{METHODS}

\section{Animal Preparations}

The institutional animal care and use committee of the State University of New York Downstate Medical Center (Brooklyn, NY) approved all the study procedures. Terminal experiments were conducted on 5 adult bonnet macaque monkeys ( 4 males and 1 female). The macaques were initially sedated by intramuscular injection of ketamine and xylazine. General anesthesia was induced and maintained using isoflurane and fentanyl with monitoring of blood pressure, oxygen saturation, end-tidal carbon dioxide, and electrocardiography. Endotracheal intubation was performed. Bilateral upper extremity intravenous (IV) catheters were inserted for administration of IV fluids and medications. An arterial line was inserted into the right arm by way of a cut down for continuous blood pressure monitoring and arterial blood gas sampling. A femoral sheath was inserted into the right femoral artery by way of a femoral cut down to provide access for catheter intervention.

\section{Animal Manipulations}

To create acute cerebral ischemia and stroke, an occlusive microcatheter (2.7F; ie, $0.9 \mathrm{~mm}$ outer diameter) was introduced by femoral access under fluoroscopic guidance and then wedged at the origin of the right middle cerebral artery (MCA). Manipulation of the catheter through the cerebral vasculature was aided by multiple iodinated IV contrast (Omnipaque 300) and verapamil injections. The microcatheter was left in place for 3 hours, followed by 1 hour of reperfusion.

\section{NIRS Imaging Setup}

Continuous functional NIRS (fNIRS) recordings were maintained throughout the experiment (before, during, and after cerebral ischemia) until the end of the reperfusion period. A continuous-wave NIRS diffuse optical tomography imager (NIRx DYNOT Compact Imager; NIRx Medical Technologies, Los Angeles, Calif; www.nirx.net [this specific product is no longer commercially available]) and a custom laboratory-made measuring head was used, sampling 2 wavelengths (760 and $830 \mathrm{~nm})$ to distinguish the hemoglobin $(\mathrm{Hb})$ oxygenation states, at a $7.94-\mathrm{Hz}$ rate, with 30 detection sites, 9 of which were co-located (coaxial fiberoptic cables) with illumination-source sites. Thus, $9 \times 30=270$ data acquisition channels were available in all (many times more channels than in currently available oximeters). The thick layer of scalp muscle covering the bonnet macaque cranium $(>3 \mathrm{~cm})$ was excised to prevent interference from extracerebral signals (this step and the subsequent one would be unnecessary in human studies, because the scalp is much thinner). The optical probe array was then attached to the exposed skull with bone screws.

\section{Processing of Optical Measures}

Because all phenomena of interest evolved much more slowly than the 7.94-Hz fNIRS sampling frequency, the raw data time series could be 
Axial
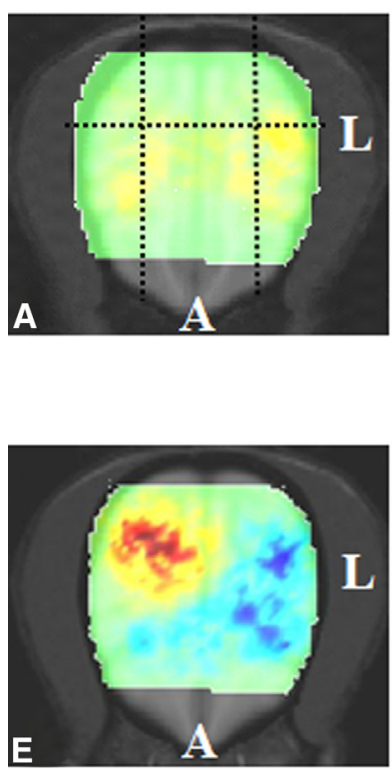

Coronal
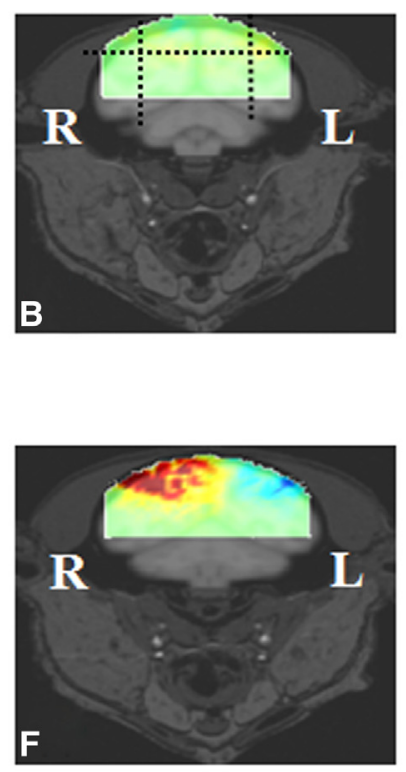

Sagittal (Left)
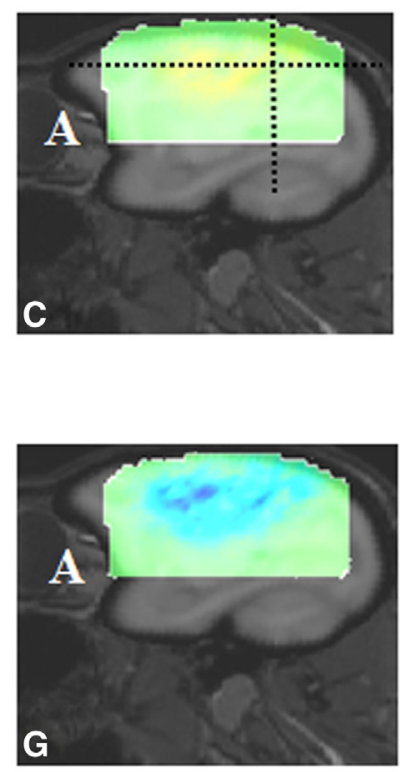

Sagittal (Right)
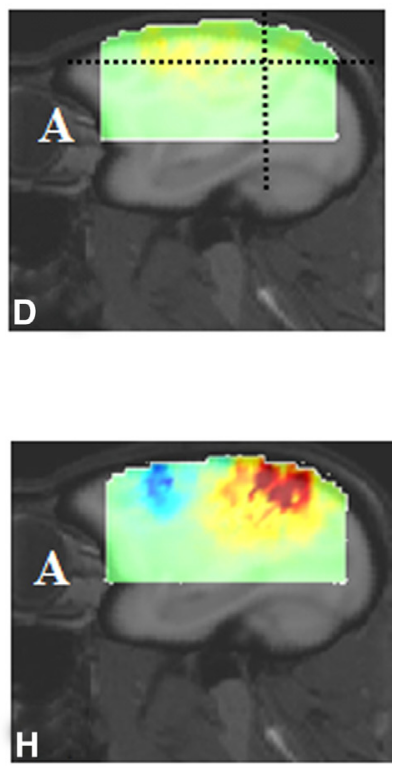

$-5.5 e-7$

0

$+5.5 \mathrm{e}-7$

FIGURE 1. Two-dimensional sections of 3-dimensional changes in oxyhemoglobin images reconstructed from data collected (A-D) before (dotted lines indicate intersections among the plotted 2-dimensional section) and (E-H) after right middle cerebral artery verapamil injection. A and E, Axial section; $\mathrm{B}$ and $\mathrm{F}$, coronal section; $\mathrm{C}$ and $\mathrm{G}$, left hemisphere sagittal section; D and $\mathrm{H}$, Right hemisphere sagittal section. $A$, Anterior; $L$, left; $R$, right. The colorbar units are moles per liter.

downsampled to $0.3-0.4 \mathrm{~Hz}$ to produce a more convenient data length, with negligible loss of information. The downsampling was accomplished by averaging blocks of adjacent data values, yielding the additional benefit of smoothing the data.

To further enhance signal quality, a low-pass filter was applied to the downsampled data $(0.1 \mathrm{~Hz})$. To correct for interchannel variations in amplifier gain and optical coupling efficiency, the filtered data for each source-detector pair were normalized to the mean value during an initial baseline interval (ie, from the start of the recording to insertion of the occluding catheter into the cerebral vasculature). ${ }^{17}$ The coefficient of variation, within the baseline time segment, was computed for each normalized data time series. Data for all source-detector pairs with a coefficient of variation of $\geq 25 \%$ (ie, noise), for either wavelength, were excluded from processing.

\section{Three-Dimensional Image Reconstruction}

The previously described normalized difference method was used to reconstruct images of oxyhemoglobin $\left(\mathrm{Hb}_{\mathrm{oxy}}\right)$, deoxyhemoglobin $\left(H b_{\text {deoxy }}\right)$, and total $\mathrm{Hb}\left(\mathrm{Hb}_{\text {total }}\right)$ from the preprocessed 2-wavelength data. ${ }^{18}$ The resulting image time series were set to a mean value of 0 during the initial baseline time segment. Thus, the images presented in Figures 1 and 2 show the $\Delta \mathrm{Hb}$ values (ie, fluctuations in $\mathrm{Hb}$ concentration about the baseline mean) and, accordingly, can have a positive or negative value. To aid the process of assigning hemodynamic image features to the anatomic structures, the images were overlaid onto a structural magnetic resonance imaging (MRI) scan of the macaque head, which was also used in the computations associated with image reconstruction. ${ }^{19}$

The normalized difference method is computationally efficient and is largely insensitive to potentially confounding factors such as intersubject variability in tissue optical coefficients. A tradeoff is that the computed image values tend to quantitatively underestimate the true $\mathrm{Hb}$ concentrations. ${ }^{20}$
However, this did not affect the accuracy of the comparisons among the image values in the different regions or at different times within a selected region.

\section{MRI Studies}

At the completion of the manipulations and NIRS recording, the macaques were transported to the imaging suite under IV anesthesia, continuous monitoring, and positive pressure ventilation by way of an endotracheal tube. Standard MRI stroke protocol scans were performed with a $1.5 \mathrm{~T}$ MRI scanner, using the standard head coil used for monkey brain imaging studies. The standard stroke protocol sequences, including diffusion, T2-weighted, T1-weighted, and fluid attenuated inversion recovery, were performed. ${ }^{21,22}$

\section{Histopathologic Examination}

The macaques were transported to the autopsy room and killed by euthanasia using IV pentobarbital. The brains were removed and fixed in $10 \%$ buffered formalin. Coronal sections were cut at approximately 5-mm intervals. Brain regions corresponding to the center of the infarct (determined from the postintervention radiologic data) and from the corresponding location on the contralateral side were submitted for routine tissue processing and paraffin embedding. Hematoxylin and eosin-stained sections, each 8 to $10 \mu \mathrm{m}$ in thickness, were then examined by light microscopy.

\section{RESULTS}

\section{Hemodynamic Response to Acute Injection}

Two-dimensional cuts of 3-dimensional images of the changes in the relative levels of oxyhemoglobin $\left(\Delta \mathrm{Hb}_{\text {oxy }}\right)$ 

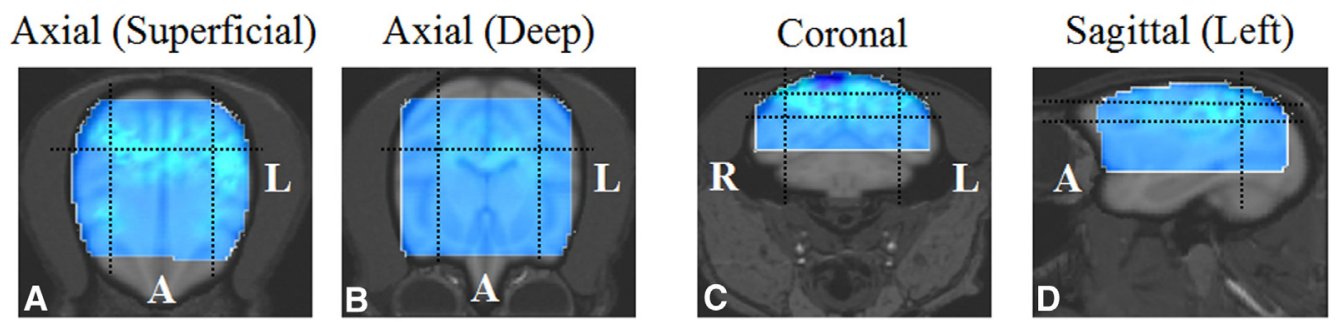

Sagittal (Right)
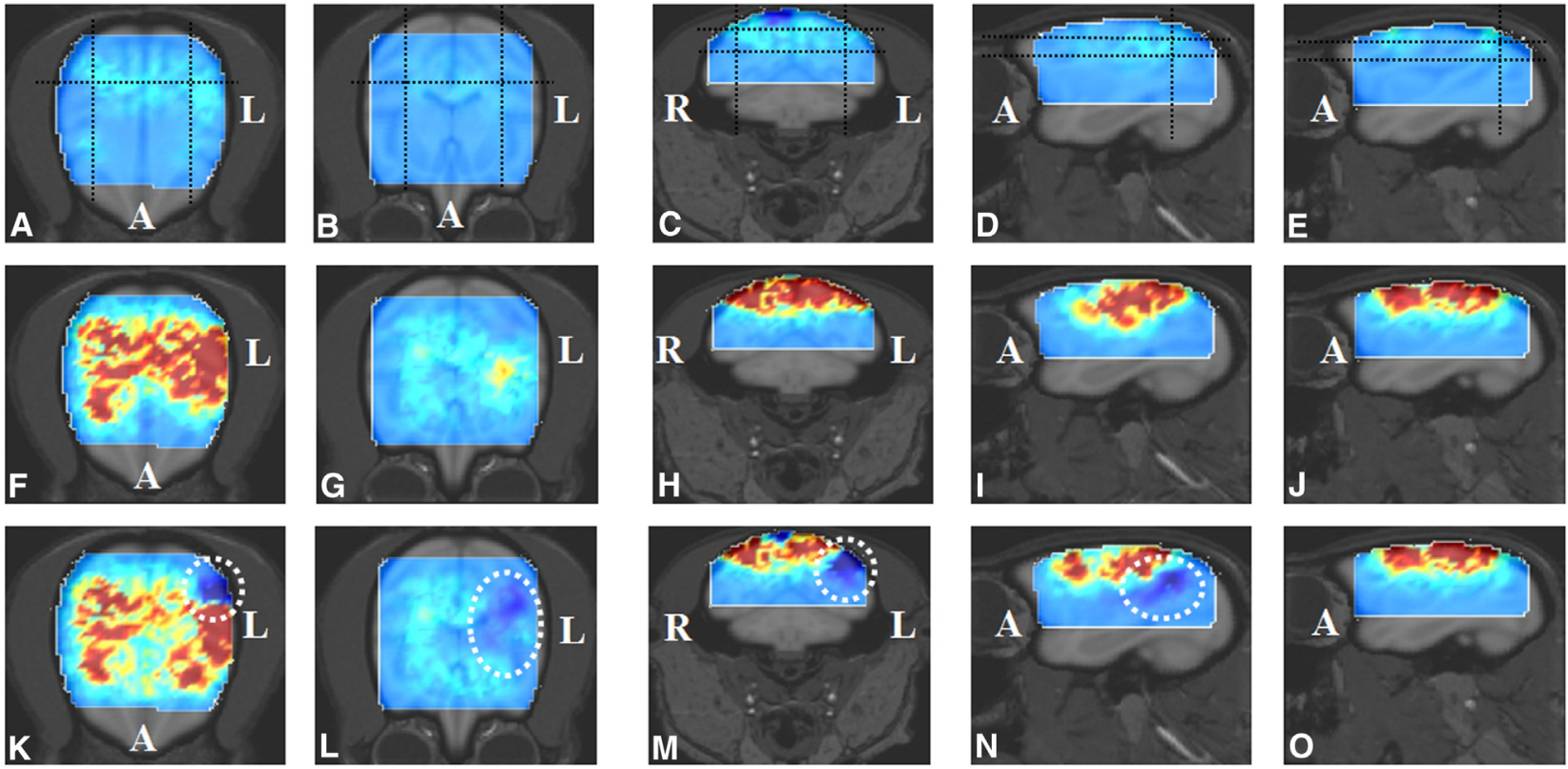

$-1.8 \mathrm{e}-7$

0

$+6.0 \mathrm{e}-7$

FIGURE 2. Two-dimensional sections of 3-dimensional change in total hemoglobin images reconstructed from data collected (A-E) before any intravascular intervention (dotted lines indicate intersections among the plotted 2-dimensional sections), (F-J) between the onset of subarachnoid hemorrhage and occlusion, and (K-O) 37 minutes after occlusion. A, F, and K, Superficial axial section; B, G, and L, deep axial section; C, H, and M, coronal section; D, I, and N, left hemisphere sagittal section; E, J, and O, right hemisphere sagittal section. K-N, Dotted circles show location of cerebral ischemia. $L$, Left; $R$, right. The color-bar units are moles per liter.

before and after injection of verapamil into the right MCA are shown in Figure 1. With all the panels of Figure 1 plotted on a common scale (ie, a given color denotes a fixed magnitude and direction of change in the $\mathrm{Hb}_{\text {oxy }}$ concentration with respect to the baseline mean value, with blue indicating a decrease and red, an increase), it can be seen that, in the images corresponding to the postverapamil injection state (Figure 1,E-H), the posterior right region distal to the injection site shows an increase in $\mathrm{Hb}_{\text {oxy }}$ compared with the preverapamil state (Figure 1, A-D). The regions of
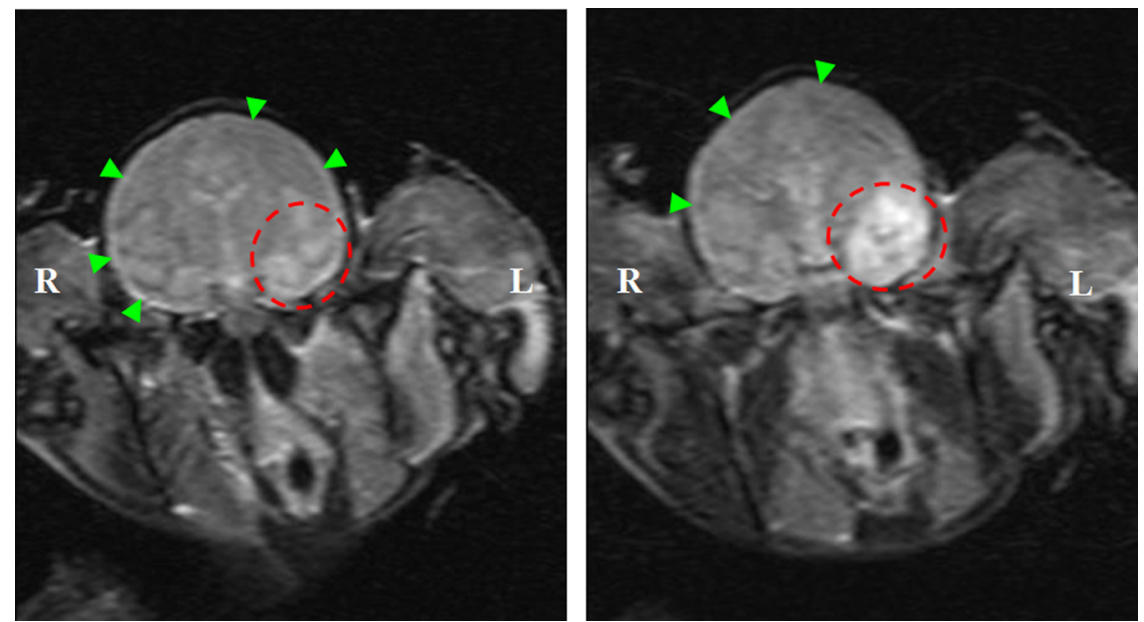

FIGURE 3. Postoperative coronal fluid attenuated inversion recovery magnetic resonance imaging sequences (slice locations: left $[L]$, right $[R]$ ) confirm onset of ischemia (dotted circle) in the left temporal and inferior frontal lobe after a period of left middle cerebral artery occlusion. The images also confirm the presence of subarachnoid hemorrhage (arrow points). 

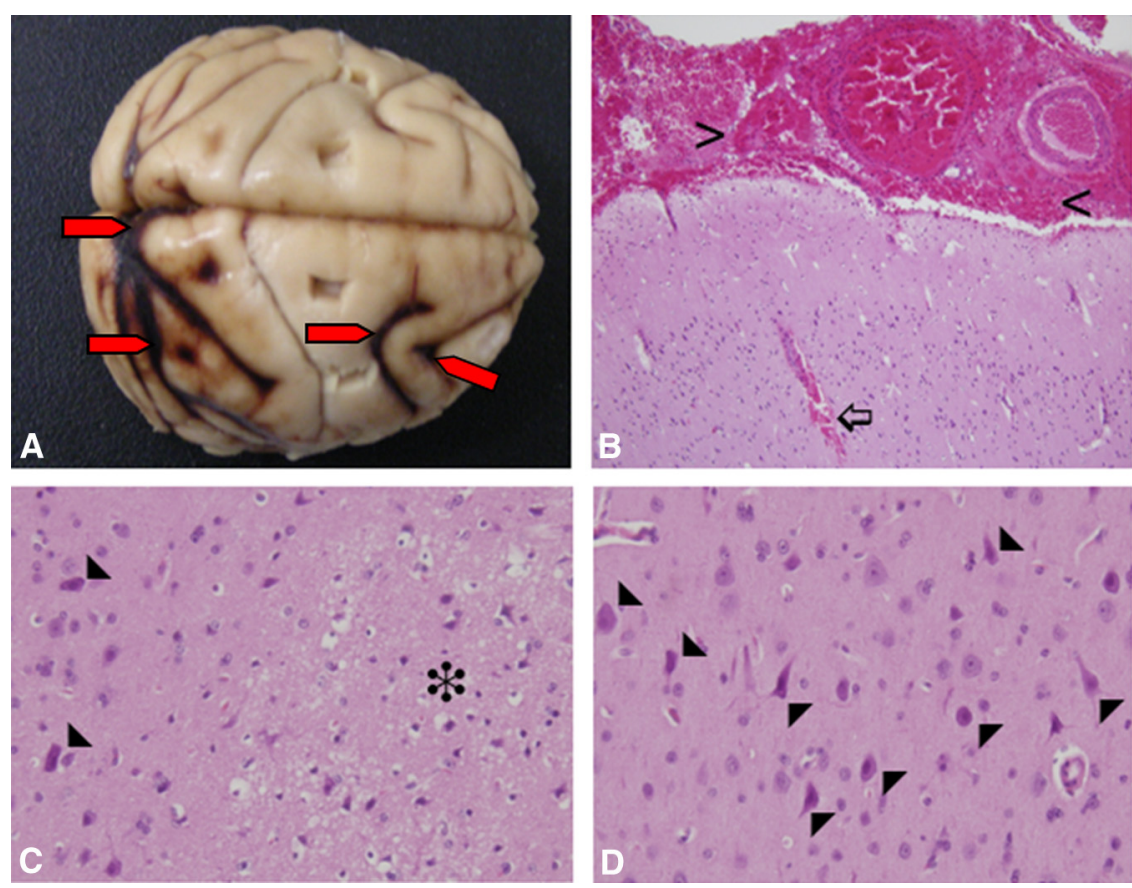

FIGURE 4. Gross pathologic and histologic findings. A, Dorsal surface of the brain, with red arrows marking subarachnoid hemorrhage present in the sulci. $\mathrm{B}$, Hemorrhage present in the subarachnoid space (chevron) and surrounding blood vessels (white arrow) (original magnification $\times 100$ ). $\mathrm{C}$ and $\mathrm{D}$, Vacuolization of neuropil (asterisk) and shrunken neurons with hypereosinophilic cytoplasm (arrowheads) indicate acute ischemic damage (original magnification $\times 400$ ). All photomicrographs were from slides stained with hematoxylin and eosin.

lowered $\Delta \mathrm{Hb}_{\text {oxy }}$ in the left and anterior right hemispheres after injection could plausibly be a result of redistribution of blood from those regions into the vasodilated (ie, lower resistance) region.

The corresponding images of $\Delta \mathrm{Hb}_{\text {deoxy }}$ and $\Delta \mathrm{Hb}_{\text {total }}$ (not shown owing to space limitations) also showed indications of verapamil-induced vasodilation (ie, $\Delta \mathrm{Hb}_{\text {deoxy }}$ decreases and $\Delta \mathrm{Hb}_{\text {total }}$ increases in the right posterior region of the brain). Thus, the combined effect was an increase in both the blood volume and the blood oxygen saturation in the tissue receiving its primary blood supply from the right MCA. Qualitatively similar results were seen in the $\Delta \mathrm{Hb}_{\text {oxy }}$ images recovered from data collected before and after injection of the iodinated angiography contrast during the procedures for catheter placement. In these cases, the primary effect was a decrease in the $\mathrm{Hb}_{\text {oxy }}$ levels on the same side of the head as the injection (of the same magnitude as the verapamil-induced increase), because the blood was diluted by the contrast.

\section{Hemodynamic Response to Cerebral Ischemia and Hemorrhage}

In 2 macaques, inadvertent bilateral internal carotid artery/MCA perforation occurred before obtaining catheter wedge occlusion of the left MCA distal to the perforation, creating striking images of bilateral subarachnoid hemorrhage (SAH) superimposed on the left-sided cerebral ischemia. Plotted in Figure 2 are 2-dimensional sections from 3-dimensional $\Delta \mathrm{Hb}_{\text {total }}$ images (the contrast between the subsequently described ischemia and hemorrhage effects is sharper in the $\Delta \mathrm{Hb}_{\text {total }}$ image than in the corresponding $\Delta \mathrm{Hb}_{\text {oxy }}$ result) reconstructed from data collected before any intravascular intervention (Figure 2, $A-E$ ) and 3.5 minutes before (Figure 2, F-J) and 37 minutes after (Figure 2, $K-O$ ) the onset of left hemisphere arterial occlusion. A clearly evident transition can be seen from a baseline $\mathrm{Hb}$ distribution similar to that shown in Figure 1, $A-D$, to one dominated by 2 phenomena: first, hemorrhage resulting from the cerebral artery perforations and corresponding increased blood volume (Figure 2, F, H-K, $M-O$ ), and, second, more localized cerebral ischemia, corresponding to a reduction in blood volume mainly in the left superior temporal gyrus (Figure 2, $K-N$ ), localized downstream of the occluded left MCA.

\section{Validation of Hemodynamic Image Findings}

The postintervention MRI scans (Figure 3) confirmed the presence of both SAH and ischemia. They also showed that the fNIRS image accurately located the ischemic region in the anteroposterior and left-right dimensions (Figure 2, $K-N$ ) and demonstrated that the ischemic region lies deeper than the hemorrhagic zone (eg, the superficial horizontal sections in Figure 2, $F$ and $K$, primarily show hemorrhage, and the deeper section in Figure 2, $L$, shows the localized 
cerebral ischemia). Additional evidence that we correctly identified the causes of the observed $\Delta \mathrm{Hb}_{\text {total }}$ changes was that hemorrhagic effects will develop rapidly and the ischemic effect takes longer to evolve. Additionally, because images recovered from back-reflection fNIRS measurements tend to underestimate the depth of $\mathrm{Hb}$ perturbations ${ }^{23}$ the trough value of the $\Delta \mathrm{Hb}_{\text {total }}$ was superficial to the MRI-determined location of the ischemic region. Although we have described a method for reducing this artifact, ${ }^{20}$ it was not applied in the present study, in the interest of minimizing computational overhead.

Gross examination showed SAH present bilaterally in the depths of the sulci and over the base of the brain. An ill-defined area of pallor and blurring of the gray-white junction was present, consistent with edema subsequent to ischemia, in the superior temporal gyrus and posterior hippocampus. Microscopic examination confirmed the presence of SAH (Figure 4, $A$ and $B$ ). Also, perivascular petechial hemorrhages were present (Figure 4, $B$ ) in the superficial cortical layers, and many of the small blood vessels appeared congested. Evidence of acute ischemic damage included focal vacuolization of the neuropil (Figure 4,C) and scattered shrunken neurons with hypereosinophilic cytoplasm and pyknotic nuclei (Figure 4, $C$ and $D$ ).

A statistical outlier analysis (Appendix E1) was used to quantify the magnitude (ie, the change in concentration for $\geq 1$ components of the $\Delta \mathrm{Hb}$ signal) and spatial extent of the 3-dimensional image features such as those shown in Figures 1 and 2. The statistical test results for the injections (either $x$ ray contrast material or vasodilator) and for hemorrhage confirmed that the resulting hemodynamic effect was mainly unilateral in the former case (contrast material, $P<10^{-4}$ [statistically significant interhemispheric differences obtained for 15 of 19 injections in all]; vasodilator, $P<10^{-7}$ ) and bilateral in the latter $\left(P<10^{-4}\right)$. Also, a highly significant interhemispheric difference was found in the numbers of pixels that become ischemic after catheter occlusion. A more detailed examination of the ischemic response demonstrated that the ischemic region showed simultaneously decreased $\Delta \mathrm{Hb}_{\text {total }}$ and increased cerebral oxygen exchange ( $\Delta \mathrm{COE}$; ie, $\left.\Delta \mathrm{Hb}_{\text {deoxy }}-\Delta \mathrm{Hb}_{\text {oxy }}\right) .^{24}$ Also, the number of image pixels with this pattern was significantly greater in the occlusion hemisphere of the brain $\left(P=10^{-5}\right)$. The average magnitude of the ischemia response was also larger in the ipsilateral hemisphere, but the interhemispheric difference was not statistically significant, owing to the small overall sample size. However, occlusion also caused a significant reduction in the number of pixels $\left(P<10^{-15}\right)$ having a simultaneously increased $\Delta \mathrm{Hb}_{\text {total }}$ and $\Delta \mathrm{COE}$ and a decreased average outlier magnitude $(P=.03)$ for those pixels.

\section{DISCUSSION}

In the present study, we have demonstrated that NIRS tomography produces real-time intraoperative functional brain images with spatial and temporal specificity in response to ischemia, vasodilatation, and hemorrhage in primates. Furthermore, the findings were validated by standard radiographic imaging and histopathologic examination. Our analysis technique, based on a modified z-score, detected outliers in real time, allowing for the definition of thresholds for immediate intraoperative intervention.

The large coverage area of our optical array ensured that if a cerebral insult were localized to a particular region, our imager would have a good chance of detecting the change in hemodynamics associated with the insult. It is understood that images reconstructed from the large-array data could be difficult to interpret in real time. Postimaging analyses, such as the z-score we have presented, would be easier to monitor by physicians in a clinical setting. Human studies are needed to identify NIRS-based metrics that correlate with subsequent neurologic dysfunction that requires intervention.

Potential barriers exist to the practical implementation of NIRS tomography in trauma or intraoperative settings. However, with advancing technological and engineering developments, many improvements in system design have been made commercially available by various manufacturers for investigational applications. ${ }^{25}$ Significant advances in the development of suitable computing environments have also been made, some of which are freely available in the public domain. ${ }^{26}$ Thus, impediments to the availability of resources for this technology are rapidly diminishing.

In addition to real-time imaging, we have previously demonstrated the feasibility of detecting and monitoring the connectivity between brain regions that share functional properties, using fNIRS. ${ }^{19}$ Assessing such functional connectivity is at the forefront of investigational protocols for understanding, diagnosis, and monitoring the treatments of various neurologic disorders. ${ }^{27}$ This capability could be a valuable asset in the prevention and management of neurologic complications of cardiac surgery, many of which extend beyond the operating room.

\section{CONCLUSIONS}

NIRS tomography is a promising modality for intraoperative functional cerebral monitoring. Additional investigations, in particular, in human subjects, are warranted.

\footnotetext{
References

1. Go AS, Mozaffarian D, Roger VL, Benjamin EJ, Berry JD, Blaha MJ, et al. Heart disease and stroke statistics-2014 update: a report from the American Heart Association. Circulation. 2014;129:e28-292.

2. Newman MF, Mathew JP, Grocott HP, Mackensen GB, Monk T, Welsh-Bohmer KA, et al. Central nervous system injury associated with cardiac surgery. Lancet. 2006 368:694-703.

3. Roach GW, Kanchuger M, Mangano CM, Newman M, Nussmeier N, Wolman R, et al. Adverse cerebral outcomes after coronary bypass surgery.
} 
Multicenter Study of Perioperative Ischemia Research Group and the Ischemia Research and Education Foundation Investigators. N Engl J Med. 1996;335: 1857-63.

4. Weintraub WS, Jones EL, Craver J, Guyton R, Cohen C. Determinants of prolonged length of hospital stay after coronary artery bypass surgery. Circulation. 1989;80:276-84.

5. Newman MF, Kirchner JL, Phillips-Bute B, Gaver V, Grocott H, Jones RH, et al. Longitudinal assessment of neurocognitive function after coronary artery bypass surgery. $N$ Engl J Med. 2001;344:395-402.

6. Phillips-Bute B, Mathew JP, Blumenthal JA, Grocott HP, Laskowitz DT, Jones RH, et al. Association of neurocognitive function and quality of life 1 year after coronary artery bypass graft (CABG) surgery. Psychosom Med. 2006;68:369-75.

7. Steinmetz J, Christensen KB, Lund T, Lohse N, Rasmussen LS. ISPOCD Group. Long-term consequences of postoperative cognitive dysfunction. Anesthesiology. 2009;110:548-55.

8. Vajkoczy P, Roth H, Horn P, Lucke T, Thomé C, Hubner U, et al. Continuous monitoring of regional cerebral blood flow: experimental and clinical validation of a novel thermal diffusion microprobe. J Neurosurg. 2000;93: 265-74.

9. Guarracino F. Cerebral monitoring during cardiovascular surgery. Curr Opin Anaesthesiol. 2008;21:50-4.

10. White H, Venkatesh B. Application of transcranial Doppler in the ICU: a review. Intensive Care Med. 2006;32:981-94.

11. Rose JC, Neill TA, Hemphill C III. Continuous monitoring of the microcirculation in the neurocritical care: an update on brain tissue oxygenation. Curr Opin Crit Care. 2006;12:97-102.

12. Smythe PR, Samara SK. Monitors of cerebral oxygenation. Anesthesiol Clin North America. 2002;20:293-313.

13. Casati A, Spreeafico M, Putzu M, Fanelli G. New technology for noninvasive brain monitoring: continuous cerebral oximetry. Minerva Anestesiol. 2006;72: 605-25.

14. Fischer GW. Recent advances in application of cerebral oximetry in adult cardiovascular surgery. Semin Cardiothorac Vasc Anesth. 2008;12:60-9.

15. Kishi K, Kawaguchi M, Yoshitani K, Nagahata T, Furuya H. Influence of patient variables and sensor location on regional cerebral oxygen saturation measured by INVOS 4100 near-infrared spectrophotometers. J Neurosurg Anesthesiol. 2003; 15:302-6.

16. Wolf M, Ferrari M, Quaresima V. Progress of near-infrared spectroscopy and topography for brain and muscle clinical applications. J Biomed Opt. 2007;12: 62104.

17. Schmitz CH, Klemer DP, Hardin RE, Katz MS, Pei Y, Graber HL, et al. Design and implementation of dynamic near-infrared optical tomographic imaging instrumentation for simultaneous dual-breast measurements. Appl Opt. 2005; 44:2140-53.

18. Pei Y, Graber HL, Barbour RL. Influence of systematic errors in reference states on image quality and on stability of derived information for DC optical imaging. Appl Opt. 2001;40:5755-69.

19. Barbour RL, Graber HL, Xu Y, Pei Y, Schmitz CH, Pfeil DS, et al. A programmable laboratory test bed in support of evaluation of functional brain activation and connectivity. IEEE Trans Neural Syst Rehabil Eng. 2012;20: 170-83.

20. Xu Y, Graber HL, Barbour RL. An image correction algorithm for functional 3D DOT brain imaging. Appl Opt. 2007;46:1693-704.

21. Merino JG, Warach S. Imaging of acute stroke. Nat Rev Neurol. 2010;6: 560-71.

22. Schellinger PD. A standardized MRI stroke protocol: comparison with CT in hyperacute intracerebral hemorrhage. Stroke. 1999;30:765-8.

23. Boas DA, Dale AM, Franceschini MA. Diffuse optical imaging of brain activation: approaches to optimizing image sensitivity, resolution, and accuracy. NeuroImage. 2004;23:S275-88.

24. Yoshino K, Kato T. Vector-based phase classification of initial dips during word listening using near-infrared spectroscopy. NeuroReport. 2012;23: 947-51.

25. Ferrari M, Quaresima V. A brief review on the history of human functional near-infrared spectroscopy (fNIRS) development and fields of application. NeuroImage. 2012;63:921-35.

26. NITRC. Available at: http://www.nitrc.org/search/?type_of_search=group\&q= optical + imaging \&sa. $x=61 \&$ sa. $y=21$. Accessed on August 25, 2014.
27. Nasiriavanaki M, Xia J, Wan H, Bauer AQ, Culver JP, Wang LV. High-resolution photoacoustic tomography of resting-state functional connectivity in the mouse brain. Proc Natl Acad Sci U S A. 2014;111:21-6.

\section{Discussion}

Dr Abe DeAnda, Jr (New York, NY). Dr Lee, I want to thank you for sharing your report with me ahead of time and congratulate you on this nice study. You have addressed a problem and have a well-designed study to try to glean out some information.

I have 2 questions. I hope you can give us further insight.

As you mentioned, what we use in the operating room now is either a 4-channel or an 8-channel NIRS system. This system presented in your study uses 270 channels. This is NIRS on steroids. Yet, most of us in this room probably do not know how to interpret what we have now at 4 channels or 8 channels, and sometimes we have an anesthesiologist who might be able to interpret what we are seeing, often after the fact. Some of the monitors will give you a breakdown (ie, printout) of the data after the fact. So my first question is, would it be reasonable that we are going to know what to do with these data intraoperatively in real time when we do not know how to do it with 4 channels?

The second one is, it is a very nice model of transient ischemic attacks and you did have the SAH in 2 cases. That is not what we are normally seeing, even when we are doing arch surgery. We are seeing somebody who postoperatively has perhaps some neurocognitive dysfunction seen only with high-grade testing when you give them a battery of tests to search for the dysfunction. It can be very, very subtle. That is because the insult can be very, very subtle. Is the fidelity or the sensitivity of this type of monitoring able to detect those small changes rather than these large changes?

Dr Lee. Thank you, Dr DeAnda, for this very insightful discussion.

Your questions relating to the ability to interpret intraoperative data and the sensitivity of this technology are germane to its potential clinical utility. It is true that the hemorrhage and ischemia that my presentation primarily focused on were large-magnitude hemodynamic perturbations, and that the hemorrhage was spatially extensive. However, this imaging technology is sensitive enough to detect hemoglobin concentration changes of just a few micromolar. In addition, it can detect them at the instrumentation's sampling rate, which was 8 images per second for these experiments, but can be even higher for more recently developed devices.

As far as whether this technology will be able to pick up on, say, subtle insults that lead to cognitive dysfunction, and whether realtime interpretation in the operating room will be possible, we will need to perform human studies to answer those questions. That will allow us to determine how successfully we can identify intraoperative "biomarkers" in the fNIRS data that correlate with subsequent neurological complications. In terms of the instrument and the technology, it is very sensitive and detects very minor, fast changes, as I've indicated. For example, as I have shown, even with a little bit of injected contrast, very obvious changes were noted, in real time.

In summary, I think that this technology is promising. In order to determine its effectiveness, we would have to do human studies comparing the preoperative and postoperative neurological and cognitive statuses, and looking at what we see in the operating room. Then we will have a systematic assessment of its utility. 


\section{APPENDIX E1}

In many cases, the ability to qualitatively examine images such as those shown in Figures 1 and 2, which our imaging algorithm can produce with a negligible time lag, will be sufficient to allow the surgical team to make decisions about when to intervene to reduce or prevent neurologic complications. However, the capability to perform quantitative assessments of the images, in or near real time, would also be advantageous. An example of such an application, which is conceptually simple but also clearly useful, would be an automated procedure for identifying any regions in the image volume that the team should pay attention to because of the magnitude of the hemodynamic changes occurring there. This task can be treated as an outlier identification problem.

An approach that is frequently used for outlier identification is to compute a quantity called the z-score: each data value (eg, in Figure 2, the $\Delta \mathrm{Hb}_{\text {total }}$ concentration at 1 specified location) is compared to the average value over the entire set (or image), and the resulting differences are expressed relative to the standard deviation for the entire set. ${ }^{\mathrm{E} 1}$ The outliers are the data values that produce large positive or large negative z-scores.

The data is time dependent in the application considered in the present study, and the conventional method of accounting for this in the z-score computation is to normalize the individual data values for a selected image time frame to the average and standard deviation for that same time frame. However, when this intuitive approach was applied to the 3 images of $\Delta \mathrm{Hb}_{\text {total }}$ in Figure 2 (ie, top, middle, and bottom row), an initially puzzling result was obtained. Although substantial and obvious differences were present among the images, similar ranges of $\mathrm{z}$-score values were obtained for all 3 (eg, in every case, we found that approximately $1 \%$ of the image pixels had z-scores greater, in absolute value, than 5.5). The reason for this is that the hemodynamic changes affect the mean and standard deviation to the same extent that they affect the individual data values, precisely because all these quantities were computed for the same image time frame.

The novel-to our knowledge-contribution that we made to the outlier identification problem was the realization that we can compare the individual pixel values for 1 measurement point (eg, after an intervention or procedure) to the mean and standard deviation for a different time (eg, before the intervention). In doing this, we identified data values that underwent large changes over time and had the potential to correlate them with the surgical events.

We also have extended the preceding 2-interval approach to situations in which the goal is to identify outliers, not on the basis of a single hemodynamic parameter, but 2 (or more) considered simultaneously. ${ }^{\mathrm{E} 2}$ In these cases, the z-score cannot be directly used as the outlier-identifying metric. Instead, we used a multivariate analog of the $\mathrm{z}$-score, known as the Mahalanobis distance ${ }^{\mathrm{E} 1}$ (Appendix Table E1).

In practice, we have found it particularly informative to take a single, range of time points from near the start of the measurement session (ie, before any major interventions) as the reference state for all z-score and Mahalanobis distance computations. An additional, practical benefit to this approach is that the mean and standard deviation need be computed only once. This allows us to consider the development of real-time display tools that will threshold image information such that only the regions having parameter values that might require a response from the surgical team are displayed. The fixed reference-interval approach was used in generating the statistical test results listed in Appendix Table E1, for which exact binomial tests ${ }^{\mathrm{E} 3}$ were used to compare the numbers of pixels (which is proportional to the size of the affected region) that are outliers in the 2 hemispheres for the various significant events that occurred during surgery.

In Appendix Table E1, the binomial test results for injections (either $\mathrm{x}$-ray contrast material or vasodilator) and for SAH confirmed that the resulting hemodynamic effect was mainly unilateral in the former case and bilateral in the latter. Also, a highly significant interhemispheric difference was found in the numbers of pixels that became ischemic after catheter occlusion. A more detailed examination of the ischemic response demonstrated that the ischemic region showed simultaneously increased $\triangle \mathrm{COE}$ and decreased $\Delta \mathrm{Hb}_{\text {total }}$. Also, the number of outliers with this pattern was significantly greater in the occlusion hemisphere of the brain. The average magnitude of the outliers was also larger in the occlusion hemisphere; however, the Mann-Whitney $U$ test result was not significant owing to the small overall sample size. Occlusion also had the secondary effect of producing statistically significant reductions in both the number (binomial test) and average outlier magnitude (unequal variance $t$ test) of the outliers with a simultaneously increased $\triangle \mathrm{COE}$ and increased $\Delta \mathrm{Hb}_{\text {total }}$.

\section{E-References}

E1. De Maesschalck R, Jouan-Rimbaud D, Massart DL. The Mahalanobis distance Chemometr Intell Lab Syst. 2000;50:1-18.

E2. Wylie GW, Graber HL, Voelbel GT, Kohl AD, DeLuca J, Pei Y, et al. Using covariations in the $\mathrm{Hb}$ signal to detect visual activation: a near infrared spectroscopic imaging study. NeuroImage. 2009;47:473-81.

E3. McDonald JH. Handbook of Biological Statistics. 2nd ed. Baltimore: Sparky House Publishing; 2009:24-32. 
TABLE E1. Statistical test results

\begin{tabular}{|c|c|c|c|c|c|}
\hline Event/intervention & Comparison & $\begin{array}{c}\text { Statistical outlier } \\
\text { metric }\end{array}$ & Image feature & Test & $P$ value \\
\hline $\begin{array}{l}\text { Contrast bolus } \\
\text { injection }\end{array}$ & Left vs right hemisphere & z-Score* & Region of $\downarrow \Delta \mathrm{Hb}_{\text {total }}$ on injection side & Binomial & $<2 \times 10^{-5} \dagger$ \\
\hline Verapamil injection & Left vs right hemisphere & z-Score & Region of $\uparrow \Delta \mathrm{Hb}_{\text {total }}$ on injection side & Binomial & $2 \times 10^{-8}$ \\
\hline \multirow[t]{3}{*}{$\mathrm{SAH}$} & Pre- vs post-SAH & z-Score & $\uparrow \Delta \mathrm{Hb}_{\text {total }}$ after onset of SAH & Binomial & $5.5 \times 10^{-5}$ \\
\hline & \multirow[t]{2}{*}{ Left vs right hemisphere } & z-score & $\uparrow \Delta \mathrm{Hb}_{\text {total }}$ in both hemispheres & Binomial & .3 \\
\hline & & $\begin{array}{l}\text { Mahalanobis } \\
\text { distance* }\end{array}$ & In both hemispheres, $\downarrow \Delta \mathrm{COE}$ and $\uparrow \Delta \mathrm{Hb}_{\text {total }}$ & Binomial & .15 \\
\hline \multirow[t]{7}{*}{ Ischemia } & \multirow[t]{7}{*}{ Left vs right hemisphere } & \multirow[t]{2}{*}{ z-Score } & Region of $\downarrow \Delta \mathrm{Hb}_{\text {total }}$ larger on occluded side & Binomial & $<10^{-13}$ \\
\hline & & & Region of $\uparrow \Delta \mathrm{COE}$ larger on occluded side & Binomial & $2 \times 10^{-5}$ \\
\hline & & \multirow{5}{*}{$\begin{array}{l}\text { Mahalanobis } \\
\text { distance }\end{array}$} & \multirow{2}{*}{$\begin{array}{l}\text { Region of } \downarrow \Delta \mathrm{Hb}_{\text {total }}+\uparrow \Delta \mathrm{COE} \text { larger on } \\
\text { occluded side }\end{array}$} & Binomial & $1 \times 10^{-5}$ \\
\hline & & & & Mann-Whitney $U$ & $\begin{array}{c}.5 \\
-10^{-15}\end{array}$ \\
\hline & & & $\begin{array}{l}\text { Region of } \uparrow \Delta \mathrm{Hb}_{\text {total }}+\uparrow \Delta \mathrm{COE} \text { is smaller on } \\
\text { occluded side }\end{array}$ & $\begin{array}{l}\text { Binomial } \\
t \text { Test }\end{array}$ & $\begin{array}{c}<10^{-15} \\
.03\end{array}$ \\
\hline & & & \multirow{2}{*}{$\begin{array}{l}\text { Region of } \uparrow \Delta \mathrm{Hb}_{\text {total }}+\downarrow \Delta \mathrm{COE} \text { larger on } \\
\text { occluded side } \ddagger\end{array}$} & Binomial & $<10^{-12}$ \\
\hline & & & & $t$ Test & .8 \\
\hline
\end{tabular}

$\uparrow$, Increased; $\downarrow$, decreased; $\triangle C O E$, cerebral oxygen exchange; $\triangle H b_{\text {total }}$, change in total hemoglobin; $S A H$, subarachnoid hemorrhage. *Discussed in Appendix E1. $\dagger$ Average value for 15 of 19 injections that showed statistically significant interhemispheric differences in the numbers of outliers. țSAH lying outside the zone of occlusion. 